\title{
Seven suggestive quantitative trait loci influence hygienic behavior of honey bees
}

Received: 15 May 2002 / Accepted: 1 October 2002 / Published online: 26 October 2002

(C) Springer-Verlag 2002

\begin{abstract}
In 1964, Walter Rothenbuhler proposed a twogene model to explain phenotypic variance in the remarkable behavior in which honey bee workers remove dead brood from their colonies. Rothenbuhler's model proposed that one locus controls the uncapping of brood cells containing dead pupae, while a second controls the removal of the cell contents. We show here, through molecular techniques and quantitative trait loci (QTL) linkage mapping, that the genetic basis of hygienic behavior is more complex, and that many genes are likely to contribute to the behavior. In our cross, we detected seven suggestive QTLs associated with hygienic behavior. Each detected QTL controlled only $9-15 \%$ of the observed phenotypic variance in the character.
\end{abstract}

\section{Introduction}

The term hygienic behavior is used to describe a two-step process carried out by some worker honey bees upon detection of dead or diseased larvae or pupae in brood comb. The process first involves 'uncapping' (elimination of the wax cap covering the brood cell), followed by 'removal' of the corpse inside (Spivak and Gilliam 1998). To explore the genetic control of this behavior, Rothenbuhler (1964) performed an elegant backcrossing experiment. First he crossed a strongly hygienic line with a non-hygienic line. The $F_{1}$ hybrid colonies were nonhygienic, and these colonies were backcrossed to the hygienic line. The backcross colonies segregated into four behavioral phenotypes, as would be expected from two

We dedicate this paper to the memory of Walter Rothenbuhler, pioneer behavioral geneticist, who passed away in August 2002.

K.L. Lapidge · B.P. Oldroyd ( $\bullet$ )

School of Biological Sciences, Macleay Building A12,

University of Sydney, NSW 2006, Australia

e-mail: boldroyd@bio.usyd.edu.au

Fax: +61-2-93514771

M. Spivak

Department of Entomology, University of Minnesota, St Paul, USA independently assorting Mendelian genes. From these data, Rothenbuhler (1964), inferred that the two components of hygienic behavior are each controlled by an independent locus. Workers that are homozygous recessive $(u u)$ at the 'uncapping' locus, uncap cells containing dead pupae whereas workers that are $U u$ or $U U$ fail to uncap dead cells. Workers that are $r r$ at the 'removal' locus remove dead pupae from uncapped cells, whereas $R r$ and $R R$ individuals do not remove dead brood. Thus expression of the behavior requires workers to be homozygous recessive at both loci (rruu).

Examples of quantitative variance in behavior and artificially induced single-gene mutants that affect the behavior of animals abound (Yamamoto et al. 1997). However, with one exception (Rinderer and Beaman 1995), also from honey bees, no naturally occurring variation in a complex behavior of a higher organism has been suggested to be under such strict genetic control as hygienic behavior of honey bees. Thus Rothenbuler's results are often cited in textbooks as one of our best examples of a complex behavior under the control of simple Mendelian genetics (e.g., Herskowitz 1977; Ridley 1995). Although Rothenbuhler's two-gene model has been challenged (Moritz 1988), it is still widely believed that hygienic behavior of honey bees is controlled by a few Mendelian genes of large affect.

\section{Materials and methods}

Crossing

To investigate Rothenbuhler's two-gene model of hygienic behavior using modern genomics we repeated his crossing scheme by instrumentally inseminating (Harbo 1986) a queen from a hygienic line with a male from a non-hygienic line (Spivak and Gilliam 1998). We then reared a queen from this cross to produce an $F_{1}$ queen. Sons of this queen should have been segregating for any loci associated with hygienic behavior.

Individual sons of this $F_{1}$ queen were then used to artificially inseminate ( $n=90$ successful inseminations) individual queens reared from the putative recessive parent (based on Rothenbuhler's model) - the hygienic queen. 
Hygienic behavior bioassays

Colonies of workers arising from the 90 backcrossed queens were then scored in a hygienic behavior bioassay (Spivak 1998; Spivak and Reuter 1998) on 1-3 separate occasions over three consecutive weeks in summer 1998 at a location north of Kurrajong, NSW, Australia. Colonies were housed in six frame nucleus colonies and were generally populous due to excellent breeding conditions during the preceding month. During the assay, there was a light nectar flow.

Each colony received two scores, one for expression of the uncapping phenotype only and one for expression of overall hygienic behavior (uncapping + removal). The scores were the proportion of about 100 dead brood cells that were uncapped or removed after 48 h (Spivak 1998; Spivak and Reuter 1998).

\section{Linkage mapping}

We extracted DNA from 119 sons of the $\mathrm{F}_{1}$ queen (including the 90 successful fathers) using a CTAB lysis method (Hunt 1997). Molecular markers were generated from these DNA samples using RAPD (Williams et al. 1990; Hunt 1997), and microsatellite loci (Estoup et al. 1993) and a sequence tagged site (Hunt et al. 1995). We also scored each male for one of two possible alleles at the allozyme locus malate dehydrogenase (Nielson et al. 1994). Combined, these analyses produced 482 scorable segregating markers.

Full multipoint linkage analysis of these 482 markers across the 119 males using the Kosambi mapping function of Mapmaker v 3.0 (Lander et al. 1987) was used to produce a new linkage map of the honey bee using methods appropriate to haplo-diploid social insects (Hunt and Page 1995; Hunt et al. 1995, 1999). A LOD score of 3.0 and a recombination fraction of 0.34 were used as the criteria for linkage.

\section{Quantitative trait loci analysis}

Complete phenotypic (i.e., successfully scored on three occasions) and molecular data were available for 63 segregating colonies. In association with the map generated from the 119 males, these data were analyzed using MapQTL v 3.0 software in order to identify any quantitative trait loci (QTLs) (Jansen 1993; Jansen and Stam 1994) associated with hygienic behavior.

\section{Results}

A numerical score based on the average level of hygienic behavior exhibited over the triplicate assays was assigned to each colony. We defined the following phenotypic classes: hygienic, $>95 \%$ of dead brood removed in $48 \mathrm{~h}$; non-hygienic, $<50$ dead brood cells removed in $48 \mathrm{~h}$; intermediate, $50-95 \%$ of dead brood removed in $48 \mathrm{~h}$ (Spivak 1998).

We assessed the repeatability of the traits uncapping and total hygiene (i.e., proportion of dead brood removed) across the three assays ( $n=90$ colonies) as a measure of the upper bound of heritability (Falconer 1981). The proportion data were first normalized using an arcsine $\sqrt{x}$ transformation. The repeatability, $r$, of uncapping was $0.54 \pm 0.061( \pm$ SE) (Becker 1984) and the repeatability of overall hygienic behavior was $0.57 \pm 0.059$.

Linkage analysis produced a linkage map of 358 marker loci over 25 linkage groups, spanning a total distance of 3,406.2 cM. The average distance between each marker was $9.5 \mathrm{cM}$. Loci that could not be successfully joined to linkage groups, or that generated unacceptably large linkage maps, were discarded. Based on this total map size and number of linkage groups, the LOD score that is declared significant for a genome-wide significance level of 5\% is 3.3 (van Ooijen 1999).

QTL analysis for overall hygienic score identified six suggestive and one significant QTL associated with linkage groups 2, 4, 5, 6, 13, and 22 (Fig. 1, Table 1). QTLs located on linkage groups 2, 4, 6, and 22 were associated with the uncapping scores only (Table 22). Two loci were in common; two were independent (Tables 1 and 2). This may suggest that QTLs linked to markers 242_0.24, 172_0.5f, 395_0.7 and G17_1.0 influence the removal aspect of hygienic behavior but not uncapping.

Individually, each QTL is of small effect, with each explaining only $9-16 \%$ of the variance observed (Tables 1 and 2). Collectively, the putative QTLs identified here
Table 1 Putative QTLs for hygienic behavior in honey bees: overall hygienic behavior

\begin{tabular}{llll}
\hline Linkage group & Nearest marker & LOD score & $\%$ Variance explained \\
\hline 2 & 336_0.71 & 2.91 & 13.1 \\
4 & $397 \_0.9 f$ & 3.04 & 14.5 \\
5 & $242 \_0.24$ & 2.07 & 8.9 \\
6 & $172 \_0.5 f$ & 1.72 & 9.3 \\
13 & 395_0.7 & 2.14 & 9.2 \\
15 & G17_1.0 & 2.17 & 9.2 \\
22 & $123 \_1.0$ & $3.37^{\mathrm{a}}$ & 15.2 \\
\hline
\end{tabular}

${ }^{\text {a }}$ Significant LOD score for a genome-wide significance level of 5\%
Table 2 Putative QTLs for hygienic behavior in honey bees: uncapping only

\begin{tabular}{llll}
\hline Linkage group & Nearest marker & LOD score & \% Variance explained \\
\hline 2 & 336_0.71 & 2.68 & 12.0 \\
4 & 397_0.9f & 3.08 & 15.8 \\
6 & U15_0.75f & 1.90 & 10.4 \\
22 & $123 \_1.0$ & 2.95 & 13.6 \\
\hline
\end{tabular}


Fig. 1 LOD Score profiles of seven QTLs controlling hygienic behavior of honey bees. Profiles are shown for overall hygienic behavior (solid line) and uncapping behavior only (gray line) in seven linkage groups. LOD scores are given on the $y$ axis. A LOD score of 1.5 was considered indicative of a linked QTL. Codes refer to the RAPD primer used to obtain the marker. Loci commencing with a numeral were obtained from the University of British Colombia. Loci commencing with a letter refer to primer kits obtained from Operon Technologies, California. Numerals after the underscore are the primer within the kit. Size polymorphisms are followed by the letter ' $f$ '. All others are presence/absence polymorphisms. The nearest marker to each putative QTL is shown in bold
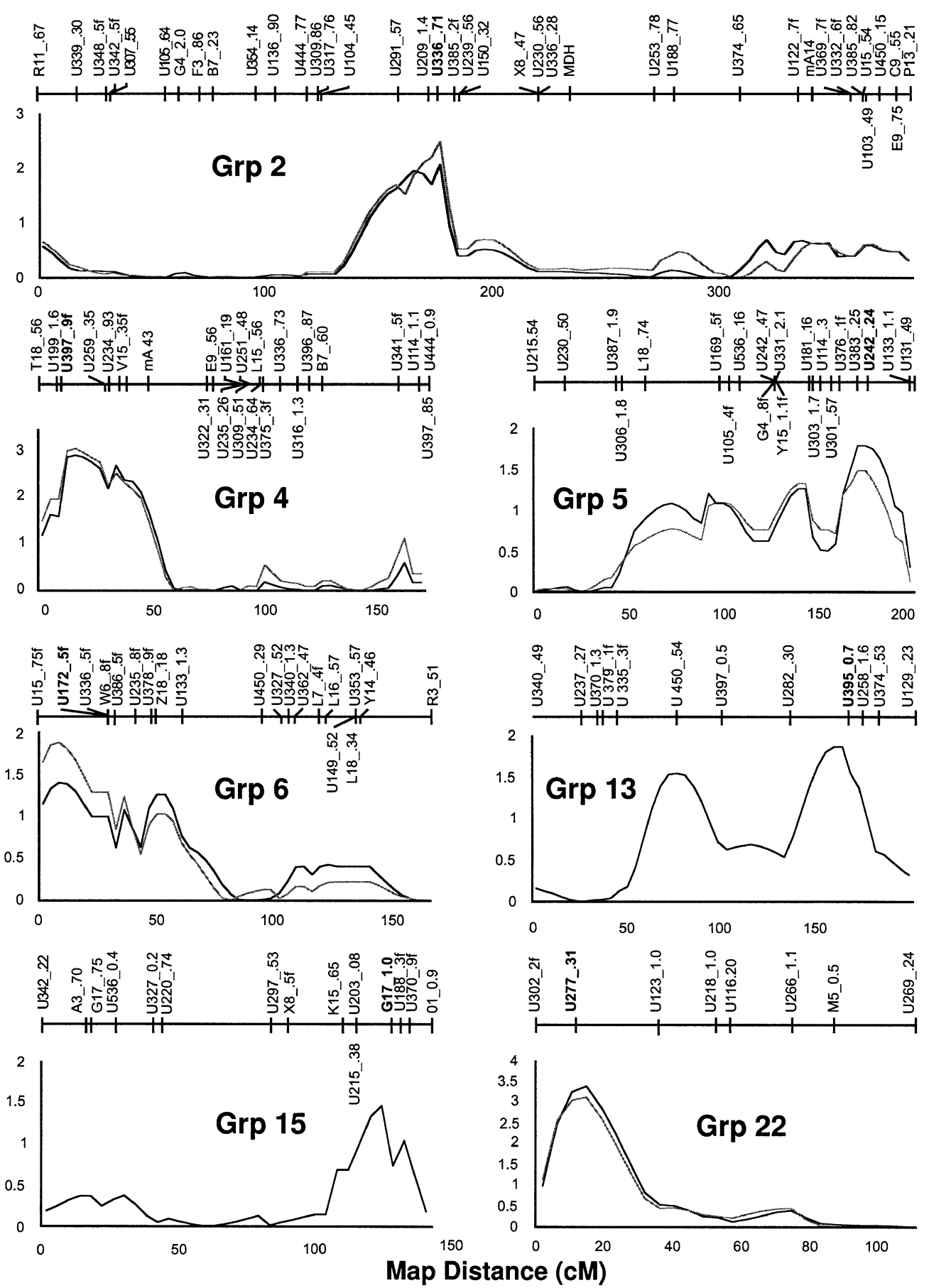

explain $79.4 \%$ of the observed variance in the expression of hygienic behavior.

\section{Discussion}

Our study revealed six markers that show suggestive linkage (van Ooijen 1999) to loci that contribute to overall phenotypic variation in honey bee hygienic behavior, and one (123_1.0) that showed strong linkage, with a significant LOD of 3.37. These markers await confirmation in independent crosses or by other means.

We expected to find two (Rothenbuhler 1964), or perhaps three (Moritz 1988), QTLs of major effect on hygienic behavior. However in our experiment we did not find any evidence for such genes. Instead we found that the behavior seems to be inherited in a more quantitative manner. Thus although our quantitative genetic analysis of repeatability and the QTL analysis confirm Rothenbuhler's findings that hygienic behavior has a strong 
genetic component (as expected by the success of selection for the character), we cannot confirm the existence of two genes of major effect that control hygienic behavior. It may be, however, that in the actual cross performed by Rothenbuhler, only two loci of large effect were segregating. Moreover, our assay is very different from that performed by Rothenbuhler. He used brood that had been naturally killed by the bacterium Paenibacillus larvae, and allowed the bees up to 16 days to remove the dead brood. The standardized test we used utilizes freeze-killed brood and is performed over $48 \mathrm{~h}$. Thus our results are not directly comparable with those of Rothenbuhler.

The number of linkage groups in a saturated map should theoretically match the number of chromosomes in an organism (Heckel 1993). The haploid number of the honey bee is 16, so the map generated in this study contains nine gaps, which are likely to span distances greater than that equating to the maximum recombination fraction of 0.34 used in the creation of the map. The map published by Hunt and Page (1995), which was also based on segregating RAPD markers, covered 3,110 cM on 26 linkage groups. Taking into account the ten gaps in their map, and a total physical size of $178,000 \mathrm{~kb}$ (Jordon and Brosemer 1974), Hunt and Page (1995) estimated the total genome size of the honey bee to be $3,450 \mathrm{cM}$, with a ratio of physical size to recombinational size of $52 \mathrm{~kb} / \mathrm{cM}$. If it is similarly assumed that the nine gaps in the map we generated are at least $34 \mathrm{cM}$ each, then we estimate the genome size to be $3,712 \mathrm{cM}$, with a ratio of physical size to recombinational size of $48 \mathrm{~kb} / \mathrm{cM}$. This size supports the claim of Hunt and Page (1995) that there is a very high recombinational rate in honey bees.

Acknowledgements We acknowledge the support and input of the Rural Industries Research and Development Corporation, Australia (RIRDC) to BPO and National Science Foundation grant IBN 9722416 to MS. Many thanks to the many beekeepers who assisted with this project, Gretchen Wheen for doing the inseminations, and Bruce White for operating the Quarantine Station efficiently. The Figure was drawn by Malcolm Ricketts. We thank the referees for very helpful comments on the manuscript.

\section{References}

Becker WA (1984) Manual of quantitative genetics. Academic Enterprises, Pullman, Wash. USA

Estoup A, Solignac M, Harry M, Cornuet J-M (1993) Characterization of (GT)n and (CT)n microsatellites in two insect species: Apis mellifera and Bombus terrestris. Nucleic Acids Res 21:1427-1431
Falconer DS (1981) Introduction to quantitative genetics, 2nd edn. Longman, London

Harbo JR (1986) Propagation and instrumental insemination. In: Rinderer TE (ed) Bee genetics and breeding. Academic Press, Orlando, Fla. pp 361-389

Heckel D (1993) Comparative linkage mapping in insects. Annu Rev Entomol 38:381-408

Herskowitz IH (1977) Principles of genetics. McMillan, New York Hunt GJ (1997) Insect DNS extraction protocol. In: Micheli MR, Bova R (eds) Fingerprinting methods based on arbitrarily primed PCR. Springer, Berlin Heidelberg New York, pp 21-24

Hunt GJ, Page RE (1995) Linkage map of the honey bee, Apis mellifera, based on RAPD markers. Genetics 139:1371-1382

Hunt GJ, Page RE, Fondrk MK, Dullum CJ (1995) Major quantitative loci affecting honey bee foraging behaviour. Genetics 141:1537-1545

Hunt GJ, Collins AM, Rivera R, Page RE, Guzman-Novoa E (1999) Quantitative trait loci influencing honeybee alarm pheromone levels. J Hered 90:585-589

Jansen RC (1993) Interval mapping of multiple quantitative trait loci. Genetics 135:205-211

Jansen RC, Stam P (1994) High resolution of quantitative traits into multiple loci via interval mapping. Genetics 148:1203-1213

Jordon R, Brosemer R (1974) Characterization of DNA from three bee species. J Insect Physiol 20:2513-2520

Lander ES, Green P, Abrahamson J, Barlow A, Daley M (1987) MAPMAKER: an interactive computer package for constructing primary genetic linkage maps of experimental and natural populations. Genomics 1:174-181

Moritz RFA (1988) A reevaluation of the two-locus model hygienic behavior in honey bees, Apis mellifera L. J Hered 79:257-262

Nielson D, Page RE, Crosland MWJ (1994) Clinal variation and selection of $\mathrm{MDH}$ allozymes in honey bee populations. Experientia 50:867-871

Ooijen JW van (1999) LOD significance thresholds for QTL analysis in experimental populations of diploid species. Heredity 83:613-624

Ridley M (1995) Animal behaviour: an introduction to behavioural mechanisms, development and ecology. Blackwell Science, Oxford

Rinderer TE, Beaman LD (1995) Genic control of honey bee dance language dialect. Theor Appl Genet 91:727-732

Rothenbuhler WC (1964) Behavior genetics of nest cleaning in honey bees. IV. Responses of $F_{1}$ and backcross generations to disease-killed brood. Am Zool 12:578-583

Spivak M (1998) Field assays for hygienic behavior in honey bees (Hymenoptera: Apidae). J Econ Entomol 91:64-70

Spivak M, Gilliam M (1998) Hygienic behaviour and its application for control of brood diseases and varroa. Bee World 79:124-134, 169-186

Spivak M, Reuter GS (1998) Honey bee hygienic behavior. Am Bee J 138:283-286

Williams JGK, Kubleik AR, Livak KJ, Rafalski JA, Tingey SV (1990) DNA polymorphisms amplified by arbitrary primers are useful as genetic markers. Nucleic Acids Res 18:6531-6535

Yamamoto D, Jallon J-M, Komatsu A (1997) Genetic dissection of sexual behavior in Drosophila melanogaster. Annu Rev Entomol 42:551-585 